\title{
Mathematical and Physical Continuity
}

\author{
Mark Colyvan \\ Department of Philosophy, \\ University of Sydney, \\ SyDNEY, NSW 2006, \\ Australia \\ mcolyvan@usyd.edu.au \\ Kenny Easwaran \\ Philosophy Program, \\ Research School of Social Sciences, \\ The Australian National University, \\ Canberra, aCt O2OO, \\ Australia \\ easwaran@gmail.com \\ Received by Greg Restall \\ Published September I6, 2008 \\ http://www .philosophy . unimelb.edu .au/ajl/2008 \\ (C) 2008 Mark Colyvan and Kenny Easwaran
}

\begin{abstract}
There is general agreement in mathematics about what continuity is. In this paper we examine how well the mathematical definition lines up with common sense notions. We use a recent paper by Hud Hudson as a point of departure. Hudson argues that two objects moving continuously can coincide for all but the last moment of their histories and yet be separated in space at the end of this last moment. It turns out that Hudson's construction does not deliver mathematically continuous motion, but the natural question then is whether there is any merit in the alternative definition of continuity that he implicitly invokes.
\end{abstract}

In his paper [2], Hud Hudson presents an interesting argument to the conclusion that two temporally-continuous, spatially-unextended material objects can travel together for all but the last moment of their existences and yet end up one metre apart. What is surprising about this is that Hudson argues that it can be achieved without either object changing in size or moving discontinuously. This would be quite a trick were it to work, but it is far from clear that it does. The problem is that Hudson's implicit notion of continuity is not the standard one. On the standardly-accepted definition of continuity, his example is straightforwardly a case of discontinuous motion. And there is no surprise that Hudson's trick can be achieved by invoking discontinuous 
motion. This, in turn, raises questions about the adequacy of the standard mathematical definition of continuity. Can a case be made for a non-standard definition of continuity and might this definition be used to support Hudson's example? We will turn to this question shortly, but first we need to see why Hudson's example relies on a non-standard definition of continuity.

We won't describe Hudson's trick in detail because there is a much simpler example that is essentially the same (which we'll get to in a moment), but the crux of Hudson's case for the motion in question being continuous is that "neither character ever moves discontinuously, for no matter how small the open region you take about the space-time point occupied by (the last part of one object) you find a temporal slice of (that object) present in that region prior to (that last time)" [2, p. 157]. Hudson's example is more complicated than it needs to be in several ways. For example, he uses extended objects in motion, rather than points, but this plays no role in the construction. We can also simplify things by talking about the function $f$ such that $f(t)$ gives the point's position at time $t$. What is crucial is that his justification of the continuity of the motion suggests that he adopts the following account of continuity: a function is continuous at a point $\mathrm{c}$ iff for every $\epsilon>0$, and for every $\delta>0$, there exists an $x \neq c$ such that $|x-c|<\delta$ and $|f(x)-f(c)|<\epsilon$ Contrast this with the now standard definition of continuity (introduced in the early I9th century by Augustin-Louis Cauchy) - a real valued function $f$ is said to be continuous at a point $\mathrm{c}$ iff for every $\epsilon>0$, there exists a $\delta>0$ such that whenever $|x-c|<\delta$, $|f(x)-f(c)|<\epsilon .[3 \text {, p. } 129]^{2}$

Perhaps the easiest way to see the difference between Hudson's account of continuity and the standard mathematical account is to consider a simpler example of a construction similar to Hudson's. Consider the function $f(x)=$ $\sin 1 / x$ for all $x \neq 0$ and $f(x)=0$ for $x=0$. This function is easily shown to be discontinuous at $x=0,3$ But in so far as we understand what Hudson has in mind by continuity, it would seem that he would admit this function as "continuous", since for any open disc around $(0,0)$ there will be some portion of the graph of $f(x)$ in that region. As we suggested, this function bears a close resemblance to Hudson's example. Indeed his example can be parametrised with respect to time $t<0$ and taking the centre of the clock in his example as

\footnotetext{
${ }^{\mathrm{I}}$ In standard mathematical terminology, this means that $\mathrm{c}$ is a limit point of (the graph of) $\mathrm{f}$. [3. 97]

${ }^{2} \mathrm{~A}$ more general definition is: for topological spaces $X$ and $Y$ a function $f: X \rightarrow Y$ is continuous just in case every open set $\mathrm{V} \subseteq \mathrm{Y}, \mathrm{f}^{-1}(\mathrm{~V}) \subseteq \mathrm{X}$ is open. [3. p. IO2]

${ }^{3}$ For $\epsilon<1$, there is no $\delta>0$ that ensures that whenever $x$ is within $\delta$ of $0, f(x)$ is within $\epsilon$ of $f(0)=0$, because $\sin 1 / x$ reaches both 1 and -1 infinitely many times in any such interval, as $1 / x$ goes off to infinity.
} 
the origin 4

$$
\begin{aligned}
& x=\frac{\sin 1 / t}{2} \\
& y=\frac{\cos 1 / t}{2}
\end{aligned}
$$

So the discontinuity of $\sin 1 / x$ at $x=0$ suffices to show the discontinuity of Hudson's example, for the former's behaviour is the same as the projection onto the $x$ axis of the latter 5

Indeed, there can be no construction that delivers what Hudson wants-at least if we restrict ourselves to the standard mathematical sense of continuity. Informally we can see the discontinuity of $f$ in the example in the last paragraph by noting that whenever $x$ is close to $0, f(x)$ need not be close to $f(c)=0$. The formal proof is in footnote 3. A similar proof shows this for any Hudson-style construction, where two continuous functions $f$ and $g$ coincide everywhere except at a single point $c$, at least one of the functions must be discontinuous at $c$. We note that by construction, $f(c) \neq g(c)$. Now take $\epsilon=|f(c)-g(c)| / 4$. This is greater than zero yet either there will be no $\delta>0$ that ensures that whenever $x$ is within $\delta$ of $c, f(x)$ is within $\epsilon$ of $f(c)$, or there will be no $\delta>0$ that ensures that whenever $x$ is within $\delta$ of $c, g(x)$ is within $\epsilon$ of $\mathrm{g}(\mathrm{c})$, because these two $\epsilon$-neighborhoods are disjoint. Thus, no version of Hudson's trick can succeed for the usual definition of continuity.

For purely mathematical purposes, the existing definition of continuity has amply proven its worth in the development of the field of analysis. There are motivations for developing other related concepts (like "uniform continuity", "differentiability", and "smoothness"), but none of these (or any other alternative) will unseat the standard account of continuity. In the metaphysics of motion, however, the situation is less clear-surely at least some actual physical objects have continuous trajectories, and it is at least possible that some physical objects move discontinuously. Thus, if (as some interpretations of quantum mechanics suggest) there is a lower bound on the size of meaningful spatial and temporal separations, then the mathematical account of continuity would not suffice for metaphysical purposes. If two distinct times can't be separated by less than $\delta$, then this value of $\delta$ would suffice to show that all possible physical trajectories are continuous. Unfortunately, Hudson's account would fail to distinguish motions as well-this same $\delta$ would show that on Hudson's account no physical trajectories are continuous. If the structure of physical space and time are different, (for instance, if one of the alternate mathematical accounts of the continuum described in [I] more accurately describes space and time,

\footnotetext{
${ }^{4}$ To make this case match Hudson's more closely, replace $1 / t$ by $-\pi \log _{2}-t$. This function takes the value $n \pi$ when $t$ is $-2^{-n}$, so each rotation around the circle takes half the time of the previous one.

5[5] also gives an example that is continuous at 0 on Hudson's definition of continuity, but clearly shouldn't be counted.
} 
using infinitesimals or some other technique) then other problems might arise for both Hudson's account and the standard account.

Regardless of what the structure of space and time are like, there might be other worries about the standard account of continuous motion. Perhaps there is an argument that trajectories like those of Hudson's objects should count as continuous motion. But even so, this wouldn't necessarily prompt a change to the specific account of continuity proposed by Hudson.

As a minimal criterion of adequacy for an account of continuity of motion in three-dimensional space (regardless of whether space and time are properly represented by real numbers), consider the following:

If an object moves continuously from point $\left(x_{A}, y_{A}, z_{A}\right)$ at time $t_{A}$ to point $\left(x_{C}, y_{C}, z_{C}\right)$ at time $t_{C}$, and $x_{B}$ is between $x_{A}$ and $x_{C}$, then there is some time $t_{B}$ between $t_{A}$ and $t_{C}$ at which the $x_{-}$ coordinate of the object's position is $x_{\mathrm{B}}$. Similarly for the $y$ - and $z$-coordinates.

(If space can't be represented by three coordinates, or if either spatial or temporal coordinates don't have an appropriate notion of betweenness, then this criterion will have to be modified.) Without some criterion like this, an object would be able to pass continuously from one side of an impenetrable barrier occupying all points with $x$-coordinate equal to $x_{B}$ to the other, which seems absurd.

The above criterion is none other than the Intermediate Value Theorem of real analysis, and can be proven to be a consequence of the standard account of continuity when space and time are real-valued. But this criterion does not entail the standard account of continuity. For example, the motion of Hudson's two objects satisfies this condition even though it doesn't satisfy the standard definition of continuity ${ }^{6}$ Thus, it allows for a meaningful debate on the appropriate metaphysical account of continuous motion. However, it still rules out Hudson's definition of continuity-as pointed out to us by Timothy Bays, if an object is at point $(0,0,0)$ at every rational time, and $(1,1,1)$ at every irrational time, then its trajectory satisfies Hudson's account of continuity, but clearly fails to meet this criterion, because it is never at any point with $x-$ coordinate equal to $1 / 2$.

This criterion also imposes further conditions if the structure of space and time is in some way "less dense" than that of the real line. If space and time are both discrete, then it imposes a sort of "speed limit"- the fastest possible motion would move one spatial unit in each dimension in every unit of time. Ad-

\footnotetext{
${ }^{6}$ To see that this motion satisfies the criterion given here, note that if $t_{A}$ and $t_{B}$ are different from 0 , then the motion from one time to the other satisfies the standard definition of continuity, and thus the Intermediate Value Theorem entails that it satisfies this criterion. But if one of these times (say $t_{A}$ ) is 0 , then we can find $t_{A}^{\prime}$ between $t_{A}$ and $t_{B}$ at which the object is at the same point as it was at $t_{A}$, and then again use the Intermediate Value Theorem to find $t_{C}$ between $t_{A}^{\prime}$ and $t_{B}$ (and therefore between $t_{A}$ and $t_{B}$ ) satisfying this criterion.
} 
ditionally, if space and time are both parametrized by rational numbers (which are "less dense" than the reals), then an object that is at point $\left(t^{2}-2,0,0\right)$ at any time $t$ violates this criterion as well-it never reaches any point whose $x$-coordinate is 0 , even though it reaches both $(-2,0,0)$ and $(2,0,0)$.

The preceding discussion suggests another way of approaching the question of finding the right account of continuity. Rather than looking at different mathematical definitions of continuity and seeing which best matches our intuitions, we might start with physical theories and see what kinds of motions the theories in question permit. We might think of these physically realistic trajectories as continuous in some sense and then seek formal mathematical descriptions of what these physically realistic motions have in common. To give an interesting example, [4] describes a case where, even without collisions, 3-dimensional Newtonian mechanics allows solutions to the $n$-body problem (for any $n \geqslant 5$ ) where a particle is ejected to infinity in a finite time. Moreover, the function (of time) describing the particle's location can be seen to be continuous at every point at which it is defined. The function has a singularity at its "exit" 7

What is interesting about this case is that if we start with the standard mathematical definition of continuity, we would say that the particle's trajectory is continuous everywhere it is defined; it's just that it is not defined at the "exit" singularity. But given that this is a physically realistic trajectory, under the approach being considered here, we might well allow that the particle's motion is continuous, period - even at the time at which it is infinitely far away. This would be hard to reconcile with the standard definition of continuity. The notion of continuity delivered by such an approach will be theory relative, in that it will depend on what kinds of motions are possible in the theory in question.

We have a couple of things to say about this approach. First, it is not at all clear why we would want to identify "physically realistic" with continuous. Surely it could be the case that some theories have it that some physically realistic motions are discontinuous-if physical theory allows for teleportation as well as ordinary movement (as in much science fiction) then surely this means that at least some discontinuous motion is physically realistic. The approach under consideration would seem to rule that out by just stipulating that any physically realistic motion will count as continuous. The second problem with such an approach (of which the first problem is just a special case) is that it does not allow intuitions about continuity to play any role. For scientific inquiry to play a role in revealing the meaning of a term, it seems that either this term must be one that belongs entirely to scientific theory (like color in quantum chromodynamics), or else there must be some pre-theoretic meaning of the term that scientific inquiry can help clarify. "Continuous" seems to be of

${ }^{7}$ Thanks to an anonymous referee for pushing us to look at the issue in this way and for drawing our attention to this example. 
the latter type, for which pre-theoretic intuitions are essential to a complete understanding of the term. Perhaps it's a more difficult case than many others, because we don't have any clear instances of actual discontinuous motion, but this just suggests that empirical investigation of the actual may be even less useful than it is in explaining the meanings of other terms, like "heat".

In any case, examples like the one described by Saari and Xia will not help rescue Hudson's trick. So it seems that while one might reasonably question the standard mathematical definition of continuity for motion in certain space-time structures, none of the plausible alternative definitions seem to push in the direction of the definition of continuity Hudson implicitly appeals to. We therefore conclude that Hudson's trick fails-at least as things currently stand. But it is important to note that the trick does not fail merely because he invokes a non-standard definition of continuity, but, rather, because there does not seem to be a plausible case to be made for the definition he relies on.

We close with a brief suggestion of how something like Hudson's advertised trick can be achieved, without changing the account of continuous motion. Two smooth ${ }^{8}$ functions can coincide over much of their domain but then part ways in such a way as to differ in value by any amount you choose over as small a non-zero interval you choose. The trick is done by using a patch function: $f(t)=0$ for all $t \leqslant 0$ and $f(t)=e^{-1 / t}$ for all $t>0$. Such functions can be used to "smooth over" a step function in an arbitrarily, but pre-specified, small region. Of course this trick isn't anywhere near as impressive as the one Hudson advertised, but it is in its spirit. And, most importantly, this trick works, and it works for the standard definition of continuity that we all know and love .9 Moreover, we suspect that this is the closest trick in the vicinity that does something like what Hudson has in mind. You can part ways smoothly but it takes some small, predetermined amount of time $\mathrm{IO}$

\section{REFERENCES}

[I] BeLL, J. 2005. The Continuous and the Infinitesimal in Mathematics and Philosophy. Monza: Polimetrica.

[2] Hudson, H. 2007. How to part ways smoothly. Analysis 67: 156-57.

\footnotetext{
${ }^{8}$ Smoothness is stronger than continuity. Smoothness involves the continuity of derivatives and, if not specified further, is usually taken to be the continuity of derivatives of all ordersso-called $\mathrm{C}^{\infty}$ functions.

${ }^{9}$ This trick is well known in mathematics circles but if you've not seen it before it is surprising that it can be achieved with a $C^{\infty}$ function. In particular, recalling Taylor's theorem from calculus, one might suppose that such a function is specified at all points by specifying its value and all derivatives at a single point, but this example distinguishes functions that are specified by their derivatives (the "analytic" functions) from the $\mathrm{C}^{\infty}$ functions.

${ }^{10}$ Thanks to Hud Hudson and Timothy Bays for discussions on the issues addressed in this paper, and to an anonymous referee of this journal for several helpful comments on an earlier draft.
} 
[3] Munkres, J. 2000. Topology (second edition). Upper Saddle River, NJ: Prentice Hall.

[4] SaARI, D.A. AND Xia, Z. 1995. Off to infinity in finite time. Notices of the American Mathematical Society 42(5): 538-46.

[5] Shackel, N. 2007. Parting smoothly? Analysis 67: 32I-24. 
The Australasian fournal of Logic (ISSN I448-5052) disseminates articles that significantly advance the study of logic, in its mathematical, philosophical or computational guises. The scope of the journal includes all areas of logic, both pure and applied to topics in philosophy, mathematics, computation, linguistics and the other sciences.

Articles appearing in the journal have been carefully and critically refereed under the responsibility of members of the Editorial Board. Only papers judged to be both significant and excellent are accepted for publication.

The journal is freely available at the journal website at

$$
\text { http://www.philosophy.unimelb.edu.au/ajl/ }
$$

All issues of the journal are archived electronically at the journal website.

SuBSCRIPTIONS Individuals may subscribe to the journal by sending an email, including a full name, an institutional affiliation and an email address to the managing editor at ajl-editors@unimelb.edu.au Subscribers will receive email abstracts of accepted papers to an address of their choice. For institutional subscription, please email the managing editor at ajl-editors@unimelb.edu.au

Complete published papers may be downloaded at the journal's website at http: //www.philosophy.unimelb.edu.au/ajl/ The journal currently publishes in pdf format.

Submission The journal accepts submissions of papers electronically. To submit an article for publication, send the $\mathrm{LT}_{\mathrm{E}} \mathrm{X}$ source of a submission to a member of the editorial board. For a current list of the editorial board, consult the website.

The copyright of each article remains with the author or authors of that article. 